\title{
Gameful Learning: Was sich aus Videospielen über Motivation lernen lässt
}

\author{
Irmlind Kammerer, Christian Fischer \\ University of Tübingen
}

Abstract: Videospiele machen süchtig, faul und gewalttätig - ist die landläufige Meinung. Neuere Erkenntnisse zeigen jedoch, dass sich diese Vorurteile nicht so leicht aufrechterhalten lassen. Im Gegenteil: Wer Videospiele spielt, kann sogar Vorteile beim Lernen haben. Mit dem Ansatz des Gameful Learning auf Plattformen wie GradeCraft soll nun auch die Motivation zum Lernen spielerisch gefördert werden. Die Erfolgschancen stehen gut.

\section{Was haben Videospiele mit dem Lernen gemeinsam?}

Egal ob am Computer oder an einer Spielekonsole: Videospiele begeistern mittlerweile seit Jahrzehnten unterschiedliche Altersgruppen und gelten als beliebter Zeitvertreib für die Freizeitgestaltung. Zu den beliebtesten Spielen gehören zweifellos diejenigen, die es verstehen, ihre Nutzerinnen und Nutzer zu fesseln, indem sie dazu motiviert werden, den nächsten Level zu erreichen und immer wieder von vorne zu beginnen, bis sich der Erfolg einstellt.

Wie würde das Lernen an Schulen und Universitäten aussehen, wenn sich dieses Erfolgsgefühl auch in den Kursen und Unterrichtsstunden vermitteln ließe? Die Realität an Universitäten sieht bisher fraglos anders aus: Studierenden steht meist nur eine begrenzte Anzahl von Versuchen offen, um eine Prüfung zu bestehen. Der konstante Leistungsdruck und die Vergleichbarkeit mit Kommilitoninnen und Kommilitonen tragen weiter dazu bei, dass der Fokus des Lernens nicht auf dem Prozess, sondern auf dem Endprodukt liegt: der guten Note.

\section{Die intrinsische Motivation in Videospielen}

Wodurch unterscheidet sich die Motivation ein Videospiel zu spielen von der Motivation zum Lernen? Und lässt sich das eine auch auf das andere übertragen, können Erkenntnisse aus der Videospielforschung also die Motivation zum Lernen fördern? Um diese Fragen zu 
beantworten wird in der Pädagogischen Psychologie häufig die Selbstbestimmungstheorie nach Richard Ryan und Edward Deci herangezogen (Ryan \& Deci, 2000). Die Selbstbestimmungstheorie unterscheidet zwischen intrinsischer und extrinsischer Motivation. Während die intrinsische Motivation dazu führt, dass eine Tätigkeit um ihrer selbst willen ausgeführt wird, weil sie der ausführenden Person etwas bedeutet, zeigen sich extrinsische Motivationsstrukturen wann immer eine Handlung als Verpflichtung wahrgenommen wird. Videospiele gehören dabei eher in den Bereich der intrinsischen Motivation, extrinsische Motive finden sich dagegen häufig in Bildungskontexten, in denen Studierende vorrangig lernen, um eine gute Note zu erreichen.

\section{Wie lässt sich die Motivation aus Videospielen auf das Lernen übertragen?}

Die gute Nachricht: intrinsische Motivation lässt sich fördern. Sowohl bei Videospielen als auch im Lernkontext spielen für die intrinsische Motivation drei Bedürfnisse eine entscheidende Rolle: das Bedürfnis nach Autonomie, nach Kompetenz und nach Zugehörigkeit.

Autonomie bezeichnet die Fähigkeit, eigene Entscheidungen treffen zu können. In Videospielen wird die Autonomie beispielsweise durch die interaktive Teilhabe gefördert. Spieler können eigene Entscheidungen treffen, die dann wiederum den Verlauf des Spiels beeinflussen. Meist wird lediglich eine grobe Richtung vorgegeben, der Weg dorthin bleibt ihren eigenen Entscheidungen überlassen. Auch die Wahl der Aufgaben, oft als „quests“ bezeichnet, und wie schnell diese bearbeitet werden, unterliegt den Entscheidungen der Spieler. Auf den Lernkontext übertragen meint Autonomie die Möglichkeit der Lernendenden, selbst darüber zu entscheiden, was, wie und in welchem Tempo sie lernen und auch in welcher Form sie eine Benotung erhalten.

Das Erleben eigener Kompetenzen wird ermöglicht, indem sowohl Spieler als auch Lernende am oberen Rand ihres Fähigkeiten-Levels abgeholt und gefordert werden. Darüber hinaus können auch unterschiedliche Schwierigkeitsstufen oder das Lösen von Aufgaben, die schwierig, aber machbar sind, zu einem Gefühl von Kompetenz beitragen. Erfolg spielt dabei 
zunächst eine untergeordnete Rolle. Durch das Erreichen des nächsten Levels etwa oder durch Ranglisten, die als zusätzlicher Ansporn dienen können, wird den Spielern ein Gefühl von Kompetenz vermittelt.

Die Zugehörigkeit zu einer Gruppe, die während eines Videospiels zusammenarbeitet und gemeinsam Punkte sammelt, kann ihren Mitgliedern das Gefühl vermitteln, Teil eines gröBeren Ganzen zu sein und somit als Ansporn dienen, als Team mehr zu erreichen. Im Lernkontext wird die Zugehörigkeit durch ein Gefühl der Verbundenheit zwischen den einzelnen Lernenden, aber auch zwischen den Lernenden und den Lehrpersonen vermittelt. Je mehr eine Tätigkeit (z.B. Videospiele spielen oder Lernen) die Bedürfnisse nach Autonomie, Kompetenz und Zugehörigkeit befriedigt, desto stärker fällt auch die intrinsische Motivation aus.

\section{Gameful Learning: Intrinsisch motiviert lernen?}

Wie sich die Erkenntnisse der Selbstbestimmungstheorie im Lernkontext umsetzten lassen, zeigen Caitlin Holman und Barry Fishman von der University of Michigan. Gemeinsam haben sie das Lernmanagement-System GradeCraft entworfen, das das Ziel verfolgt, das Autonomieempfinden, Kompetenzerleben und Zugehörigkeitsgefühl von Studierenden in Hochschulkursen zu stärken (Holman, Aguilar, \& Fishman, 2013). GradeCraft kann auf unterschiedlichste Weise eingesetzt werden und somit kann auch die Herstellung von Autonomie, Kompetenz und Zugehörigkeit jeweils durch unterschiedliche Elemente erreicht werden.

So können in einem Kurs eine Vielzahl an Aufgaben, zum Beispiel in Form von Blogoder Forumsbeiträgen, Quizzen oder der Teilnahme an Kursen ausgeführt werden, die den Lernenden „Erfahrungspunkte“ einbringen, die wiederum in eine Gesamtnote umgerechnet werden. Dabei können Lernende im Verlauf des Kurses weitaus mehr Punkte sammeln als für die bestmögliche Note notwendig sind. So können wahlweise auch Aufgaben ausgelassen werden. Eine schlechte Beurteilung einzelner Aufgaben hat nur einen geringen Einfluss auf die Endnote, da durch den Überschuss an zu erreichenden Punkten die ,,verlorenen“ Punkte wieder aufgeholt werden können. 
Die Studierenden haben somit „freedom to fail“ - sie können Risiken eingehen und haben ihr Lernen selbst unter Kontrolle. Diese Elemente zielen darauf ab, das Autonomieempfinden und Kompetenzerleben der Studierenden zu stärken. Des Weiteren sollen Ranglisten ein Gefühl der Zugehörigkeit zu einer größeren Lerngemeinschaft fördern (optional und anonym). Auch die Einteilung der Studierenden in „Häuser“, für die sie über das Semester hinweg durch verschiedene Herausforderungen Hauspunkte sammeln können, vermittelt ein Gefühl von Zugehörigkeit.

GradeCraft stellt zudem einen „Grade Predictor“ bereit, mit dem die Studierenden ihre Leistung überprüfen und mit dessen Hilfe sie einsehen können, wie viele Punkte sie schon erreicht haben, wie viele ihnen noch fehlen, um eine bestimmte Note zu erreichen und durch welche Aufgaben sie diese Punkte erhalten können. Den „Grade Predictor“ können auch die beteiligten Dozierenden einsehen und auf diese Weise führzeitig abschätzen, in welchen Bereichen Studierende mehr Förderung benötigen und ihnen ein direktes Feedback geben.

\section{Plattformen wie GradeCraft fördern die intrinsische Motivation}

Studien, die sich mit dem Erfolg von Gameful-Learning-Plattformen wie GradeCraft beschäftigen zeigen, dass sich für die Mehrheit der Studierenden durch das LernmanagementSystem positive Effekte auf erlebte Kontrolle über den eigenen Lernprozess in Kursen, den erbrachten Aufwand und das erfolgreiche Absolvieren des Kurses ergeben (z.B. Aguilar et al., 2014; Fishman \& Aguilar, 2012). Das Gameful Learning trägt somit tatsächlich dazu bei, die intrinsische Motivation zu fördern: Den Studierenden wird ein Gefühl von Kontrolle über ihre finale Note vermittelt, wodurch sie - wie bei einem guten Videospiel - eher motiviert sind, mehr Zeit in den eigenen Lernprozess zu investieren. Durch die Möglichkeit, verlorene Punkte wiedergutzumachen und die eigene Note kontinuierlich zu verbessern, sinkt zudem der Leistungsdruck und der damit einhergehende Stress. 
Das Gameful Learning macht sich somit Erkenntnissen aus der Motivationsforschung und Vorteile aus der Videospielgestaltung zunutze, um die intrinsische Motivation auch im Lernkontext zu fördern und auf diese Weise zu einem effektiveren Lernen beizutragen.

\section{References}

Aguilar, S., Karabenick, S., Fishman, B., \& Holman, C. (2014). Supporting Students’ Autonomy through Gameful Course Design. Poster presented at the International Conference on Motivation. Helsinki, Finland

Fishman, B., \& Aguilar, S. (2012). Gaming the Class: Using a Game-based Grading System to Get Students to Work Harder... and Like It. In C. Martin, A. Ochsner, \& K. Squire (Eds.), Proc. GLS 8.0 (pp. 111-118). Pittsburgh, PA: ETC Press

Holman, C., Aguilar, S., \& Fishman, B. (2013). GradeCraft: What Can We Learn From a Game-Inspired Learning Management System? Design brief for the 3rd Annual Conference on Learning Analytics and Knowledge. Leuven, Belgium.

Ryan, R. M., \& Deci, E. L. (2000). Self-determination theory and the facilitation of intrinsic motivation, social development, and well-being. American Psychologist, 55(1), 68-78.

Irmlind Kammerer, B.A., studiert Empirische Bildungsforschung und Pädagogische Psychologie an der Eberhard Karls Universität Tübingen.

Christian Fischer, Ph.D. ist Tenure-Track-Professor für Educational Effectiveness am HectorInstitut für Empirische Bildungsforschung der Eberhard Karls Universität Tübingen. 